\title{
Characteristics and Outcomes of COVID-19 Patients Admitted in the Medical ICU of a Tertiary Public Hospital in the Philippines during the First Two Months of being a COVID-19 Referral Center
}

\author{
Aprille Anne O. Octaviano, MD, ${ }^{1}$ Blessie Marie B. Perez, $\mathrm{MD}^{1}$ and Jubert P. Benedicto, $\mathrm{MD}^{2}$ \\ ${ }^{1}$ Department of Medicine, Philippine General Hospital, University of the Philippines Manila \\ ${ }^{2}$ Division of Pulmonary Medicine, Department of Medicine, Philippine General Hospital, University of the Philippines Manila
}

\begin{abstract}
Introduction. The University of the Philippines-Philippine General Hospital (UP-PGH) began its operations as a COVID-19 referral center on March 30, 2020. Local studies reporting characteristics of patients in the intensive care units (ICUs) are lacking.

Objectives. 1) To describe the baseline characteristics and outcomes of the initial cohort of COVID-19 patients in the medical ICU. 2) To report the initial situation and strategies in the ICU during the first two months of being a COVID-19 referral center.
\end{abstract}

Methods. We conducted a review of records of all patients with confirmed COVID-19 admitted to the medical ICU of UP-PGH between April 1 to May 31, 2020. Patient demographics, comorbidities, APACHE-II score, signs and symptoms, laboratory and radiologic results, respiratory and vasopressor support, and outcomes were collected.

Results. Out of 35 patients with confirmed COVID-19, majority were above 60 years old (63\%). Hypertension was the most frequent comorbidity (77\%). Fever was the most common symptom (51\%). The mean duration of symptoms prior admission was $9 \pm 7$ days. Anemia and leukocytosis with neutrophilia was common. Lactate dehydrogenase (LDH) and high-sensitivity C-reactive protein (HSCRP) were elevated in most patients. Majority of patients (66\%) had moderate level of hypoxemia on admission. Bilateral pneumonia on chest radiograph was found in 34 patients. Hydroxychloroquine and chloroquine were the most commonly used drugs. A total of 24 patients (69\%) required invasive mechanical ventilation while 15 (43\%) needed pressor support. Twenty-two expired (63\%) while thirteen (37\%) patients were discharged from the medical ICU.

Conclusion. The clinical characteristics in our set of patients are consistent with other studies on critically ill patients. Mortality in the medical ICU was high.

Key Words: coronavirus, COVID-19, ICU, Philippines

\section{INTRODUCTION}

The epicenter of COVID-19 pandemic was traced to Wuhan, China, where cases of pneumonia of unknown etiology first emerged. These were ultimately traced to a

Corresponding author: Aprille Anne O. Octaviano, MD Department of Medicine Philippine General Hospital University of the Philippines Manila Taft Avenue, Manila 1000, Philippines Email: aprilleoctaviano@gmail.com novel coronavirus (2019-nCOV), with its associated disease, coronavirus disease 2019 (COVID-19). ${ }^{1}$ Since then, it has spread to almost every region in the world and was declared a global health pandemic. The disease was observed to rapidly progress to a critical illness state which mainly caused the 
surge of hospital admissions and increased need for critical care. In a pandemic, the ability to adequately allocate critical care resources should be a primary consideration for all healthcare systems. Published studies on the clinical characteristics and outcomes of COVID-19 patients in the Philippines are still scarce, especially those admitted to intensive care units (ICUs). This information can provide important insights for decision-making regarding critical care capacity and allocation of resources.

On January 31, 2020, the first confirmed case of COVID-19 was reported in the Philippines, with the first local transmission declared on March 5, 2020. ${ }^{2}$ The University of the Philippines-Philippine General Hospital (UP-PGH), a tertiary public hospital and designated national university hospital, was delegated as one of the three COVID-19 referral centers in the Philippines, prioritizing admission of patients who were moderately- to critically-ill with COVID-19. On March 30, 2020, the national university hospital began its operations as a COVID-19 referral center ${ }^{3}$ with a total of 2,244 admissions as of November 23, 2020.

Engineering and manpower logistics were put in place in preparation for hospital operations for appropriate handling of COVID-19 patients and protection of the hospital workforce. A COVID Clinical Pathway was instituted for patients suspected of having the disease which also underscored the infection control measures to be taken by the healthcare worker. Two hospital wards and one medical ICU were allocated for confirmed COVID-19 charity patients. From previously being able to accommodate a total of 12 patients in the medical ICU, capacity was downsized to 6 beds to ensure adequate physical distancing among patients.

Knowledge of disease epidemiology is crucial to the medical community and government in planning efforts to address this pandemic. The aim of our study is to describe the demographic characteristics, comorbid medical conditions, pertinent initial signs and symptoms, laboratory and radiologic results, and outcomes of COVID-19 patients admitted at the medical ICU in UP-PGH during the first two months of being a COVID-19 referral center. We also present a report on the initial situation and initial management practices in the ICU as one of the first referral centers in the country. To our knowledge, this is the first study in the Philippines documenting the clinical characteristics and outcomes of COVID-19 patients admitted in the ICU and reporting on the initial situation and strategies as a referral center. The data collected can serve as a good baseline for subsequent pandemic responses and can also help guide other hospitals when tapped to become a referral center.

\section{METHODS}

\section{Study Design, Duration, and Participants}

All consecutive patients at least 18 years old, with laboratory-confirmed SARS-CoV-2 infection (real-time reverse transcriptase-polymerase chain reaction, RT-PCR) admitted at the medical ICU of UP-PGH from April 1 to May 31, 2020 were included in this retrospective observational study.

Ethics committee approval was obtained from the University of the Philippines Manila Research Ethics Board (UPMREB) prior to implementation of this study (UPMREB Code 2020-404-01).

\section{Testing for COVID-19}

Testing for COVID-19 was done via submission of nasopharyngeal swab samples to the Molecular Research Laboratory (MRL) of the Department of Medicine, UPPGH. All specimens were tested using RT-PCR for SARS-CoV-2. Patients were tested upon admission to the emergency department of the UP-PGH. No routine repeat testing was done upon ICU admission unless ordered by the Infectious Disease Service. Patients from other hospitals with documented COVID-19 infection requesting accommodation to the ICU were directly admitted to the medical ICU via coordinated transfer. No immediate repeat RT-PCR for SARS-CoV-2 infection was done on admission for transferred patients unless advised by the Infectious Disease Service who were part of managing these patients.

\section{COVID Clinical Pathway}

A COVID Clinical Pathway form developed by the Infectious Disease service was provided for each patient suspected of the disease, where the admitting physician can easily indicate necessary interventions required. A checklist of diagnostic tests was included in the form where baseline laboratories and chest $\mathrm{x}$-ray can be ordered. The following baseline laboratory tests were ordered for all patients: complete blood count, serum creatinine, BUN, AST, ALT, serum electrolytes ( $\mathrm{Na}, \mathrm{K}, \mathrm{Ca}, \mathrm{Mg}), \mathrm{ABG}, \mathrm{LDH}$, ferritin, lactate, high-sensitivity C-reactive protein (HSCRP), IL-6, troponin I, D-dimer, PT/PTT, and fibrinogen. Additional diagnostic tests included 12L ECG and cultures (blood culture, sputum/endotracheal aspirate culture). Highresolution computed tomography of the chest was requested upon recommendation of the Pulmonary Medicine specialty service.

During the months of April to May 2020, investigational therapies that were available and were offered to patients were the following: hydroxychloroquine, chloroquine, lopinavir-ritonavir, tocilizumab, remdesivir, convalescent plasma therapy, and hemoperfusion. Administration of the drugs and convalescent plasma therapy and hemoperfusion therapies were decided through designated multidisciplinary conferences, upon agreement among subspecialty services (i.e., Pulmonary Medicine, Infectious Disease, Hematology, Nephrology) after obtaining consent from the patient or relatives. The baseline laboratory tests requested also served as a guide to direct appropriate management and investigational therapies of the patients. 


\section{Admission to the Medical ICU}

A physician consultant served as the medical ICU team captain per week. The team captain receives endorsements and referrals from various specialties regarding critical patients with positive RT-PCR for SARS-CoV-2, including requests for transfer from other hospitals. Given the limited capacity of beds in the medical ICU, admission of a patient was based on the discretion of the team captain. Patients usually prioritized included those who have the severe and critical category of COVID-19 and those who were intubated and in shock, regardless of indication for such interventions, whether directly or indirectly from COVID-19. Patients needing critical care were accepted whether they needed medical, surgical, or neurologic care.

\section{Data Collection}

Clinical data was collected through review of available medical records, including retrieval of laboratory and radiologic results in the hospital's laboratory and imaging portals. Collection and transcription of data was done by the investigators. Authority to access data was coursed through the section of Medical Records section of the institution. The extracted data per patient included the following: age, sex, medical comorbidities, date of admission to UPPGH and medical ICU, APACHE II scores, pertinent presenting signs and symptoms, laboratory and radiologic results, modes of respiratory support (invasive mechanical ventilation, noninvasive mechanical ventilation, oxygen mask) and vasopressor support, and outcomes (mortality, discharge from medical ICU). Laboratory tests included complete blood count, inflammatory markers (lactate dehydrogenase, ferritin, high-sensitivity $\mathrm{C}$-reactive protein, procalcitonin), $\mathrm{PaO}_{2} / \mathrm{FiO}_{2}$ ratio based on arterial blood gas, and serum creatinine and electrolytes (sodium, potassium). Radiologic results were limited to chest radiographs and computed tomography of the chest, if available. All patients were followed up to discharge from ICU or death, which are the primary outcomes of this study.

\section{Statistical Analysis}

No statistical sample size calculation was performed a priori, and the sample size was equal to the number of patients admitted and treated in the medical ICU during the study duration. Descriptive statistics was used to summarize the data. Continuous variables were summarized as means \pm standard deviation (SD) and range; categorical variables were presented as counts and percentages. For any missing data, no imputations were done.

\section{RESULTS}

\section{Baseline Clinical Characteristics}

A total of 35 patients confirmed to have COVID-19 were admitted to the medical ICU of the UP-PGH from April 1, 2020 to May 31, 2020. Out of the 35 patients, 24
(69\%) were male. Mean $( \pm \mathrm{SD}$ ) age was $62 \pm 15$ (range, 20 to 81 ), with $57 \%$ of patients in the $61-$ to 80 -year-old bracket (Figure 1). Other baseline clinical characteristics of all patients are presented in Table 1.

The most common chronic medical condition was hypertension (27/35 or 77\%), followed by type 2 diabetes mellitus (11 or $31 \%$ ), chronic kidney disease (6 or $17 \%)$, and previous cerebrovascular accident (4 or 11\%). Two patients (6\%) had history of pulmonary tuberculosis and subsequent treatment and two other patients (6\%) had a diagnosis of chronic obstructive pulmonary disease. Other noted medical comorbidities include: chronic hepatitis B infection, malignancy (cervical cancer, endometrial cancer, breast cancer), bronchial asthma, and previous myocardial infarction.

The most common symptom reported on admission was fever (51\%), followed by cough (16\%) and shortness of breath (16\%). Diarrhea and nausea and vomiting was reported in 4 (11\%) and 2 (6\%) patients, respectively. Sore throat, malaise, abdominal pain were reported in a few patients. A total of 5 patients (14\%) were admitted to the medical ICU for cerebrovascular event (stroke) who were found to be positive for COVID-19. The mean $( \pm \mathrm{SD})$ duration of symptoms prior admission was $9 \pm 7$ days (range, $<24$ hours to 30 days).

Table 1. Baseline clinical characteristics

\begin{tabular}{|c|c|}
\hline Clinical Characteristic & $\begin{array}{l}\text { No. of patients (\%) } \\
(\mathrm{N}=35)\end{array}$ \\
\hline \multicolumn{2}{|l|}{ Gender } \\
\hline Male & $24(69)$ \\
\hline Female & $11(31)$ \\
\hline Age, mean (range) in years & $62 \pm 15$ (20 to 81$)$ \\
\hline \multicolumn{2}{|l|}{ Chronic medical condition } \\
\hline Hypertension & $27(77)$ \\
\hline Type 2 diabetes mellitus & $11(31)$ \\
\hline Chronic kidney disease & $6(17)$ \\
\hline Previous cerebrovascular accident & $4(11)$ \\
\hline Malignancies & $3(9)$ \\
\hline Cervical cancer & $1(3)$ \\
\hline Endometrial cancer & $1(3)$ \\
\hline Breast cancer & $1(3)$ \\
\hline Pulmonary tuberculosis & $2(6)$ \\
\hline Chronic obstructive pulmonary disease & $2(6)$ \\
\hline Bronchial asthma & $1(3)$ \\
\hline Previous myocardial infarction & $1(3)$ \\
\hline \multicolumn{2}{|l|}{ Symptoms } \\
\hline Fever & $18(51)$ \\
\hline Cough & $16(46)$ \\
\hline Shortness of breath & $16(46)$ \\
\hline Diarrhea & $4(11)$ \\
\hline Nausea and vomiting & $2(6)$ \\
\hline Stroke presentation & $5(15)$ \\
\hline Symptom duration, mean (range) in days & $\begin{array}{l}9 \pm 7(<24 \text { hours } \\
\text { to } 30 \text { days })\end{array}$ \\
\hline \multicolumn{2}{|l|}{ Vital signs on admission } \\
\hline Heart rate, mean (range), in counts per minute & $92.6 \pm 17$ (56 to 130$)$ \\
\hline Mean Arterial Pressure, mean (range) & $94 \pm 13(65$ to 127$)$ \\
\hline GCS mean (range) & $10 \pm 5$ (3 to 15$)$ \\
\hline $\begin{array}{l}\text { Respiratory rate, mean (range), in counts } \\
\text { per minute }\end{array}$ & $25 \pm 6$ (17 to 40$)$ \\
\hline Temperature, mean (range), in degrees Celsius & $37 \pm 0.7$ (36 to 40$)$ \\
\hline
\end{tabular}


During the first 24 hours of ICU admission, the mean heart rate $( \pm \mathrm{SD})$ was $93 \pm 17$ (range, 56 to 130), with 12 patients $(34 \%)$ noted to have tachycardia $(\mathrm{HR}>100)$. Mean arterial pressure $( \pm \mathrm{SD})$ was $94 \pm 13$ (range, 65 to 127 ); all patients were received with MAP $>65$, either on or off pressor support. GCS range for all patients was 3 to 15, with a total of fifteen patients (15) with a perfect score on the scale. A total of 24 patients $(69 \%)$ were received tachypneic $(R R>20)$. The mean respiratory rate $( \pm \mathrm{SD})$ on admission was $25 \pm 6$ (range, 17 to 40). Only three (3) patients were received febrile $\left(37.8^{\circ} \mathrm{C}\right.$ and above); while all the remaining patients were afebrile. The mean temperature value $( \pm \mathrm{SD})$ was $37^{\circ} \mathrm{C}$ \pm 0.7 , with range $36^{\circ} \mathrm{C}$ to $40^{\circ} \mathrm{C}$.

APACHE II scores of patients within 24 hours of admission ranged from 4 to 42 , with an average score $( \pm S D)$ of $19 \pm 9$. (Figure 2 )

\section{Laboratory Findings}

Most patients presented with anemia (60\%), leukocytosis (65\%), and neutrophilia (88\%). Among the inflammatory markers, LDH and HSCRP were found to be consistently elevated among patients. Thirty-four patients (97\%) had elevated LDH levels, while 28 (80\%) had elevated HSCRP. Only 16 (45\%) had elevated ferritin levels and 15 (43\%) had elevated procalcitonin levels. Elevation of serum creatinine consistent with acute kidney injury was seen in 16 patients (45\%). Serum sodium and potassium was within normal reference range for majority of patients $(54 \%$ and $65 \%$, respectively). Majority of patients (66\%) had moderate hypoxemia on admission.

\section{Radiologic Findings}

Chest radiographs of all patients admitted in the medical ICU showed bilateral pneumonia, except for one, where no active lung infiltrates were found. Reticulonodular opacities (8 patients), and alveolo-interstitial opacities (7 patients) were the most common findings. Other findings include: inhomogenous, airspace, and hazy opacities. In twelve patients, opacities were noted to be more pronounced in the peripheral regions.

A high-resolution computed tomographic scan (HRCT) of the chest was performed in two patients with consideration of pulmonary embolism. Both imaging findings showed ground glass opacities diffusely scattered bilaterally with

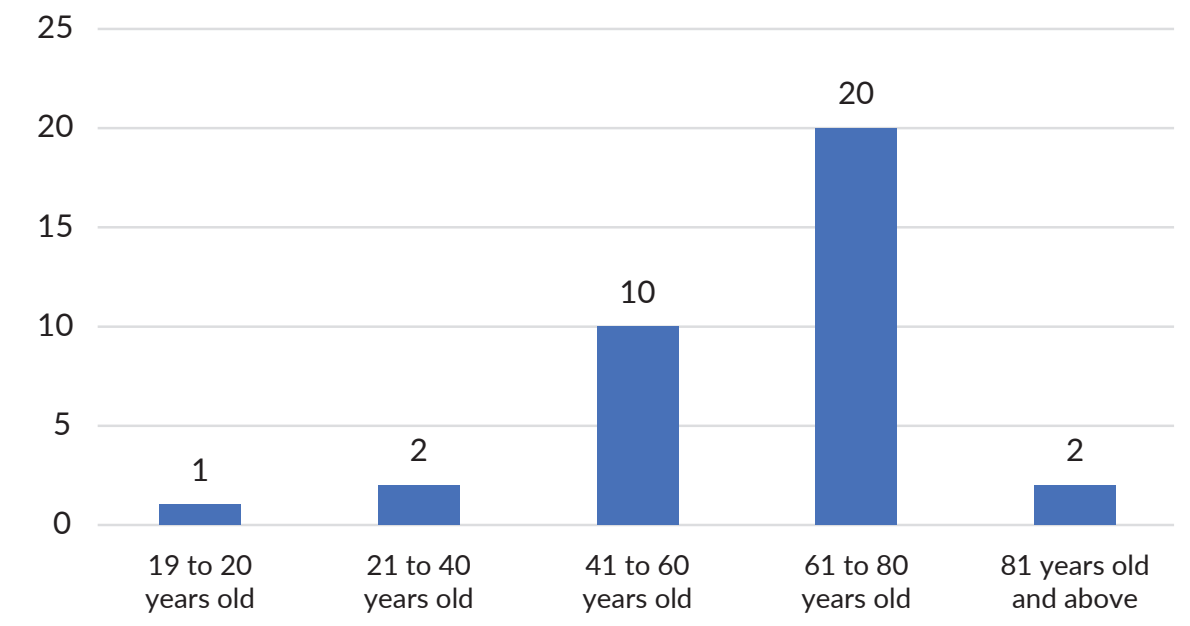

Figure 1. Age group distribution of patients with COVID-19 admitted in the medical ICU of UP-PGH.

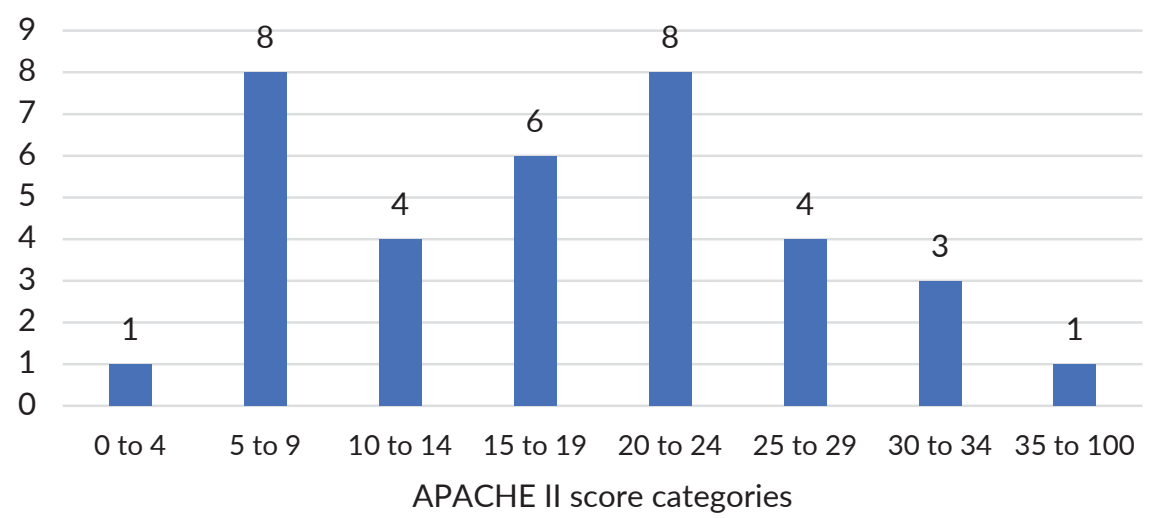

Figure 2. Distribution of patients according to APACHE II score.

Scoring system taken from Knaus et al. ${ }^{4}$ 
Table 2. Laboratory parameters on admission

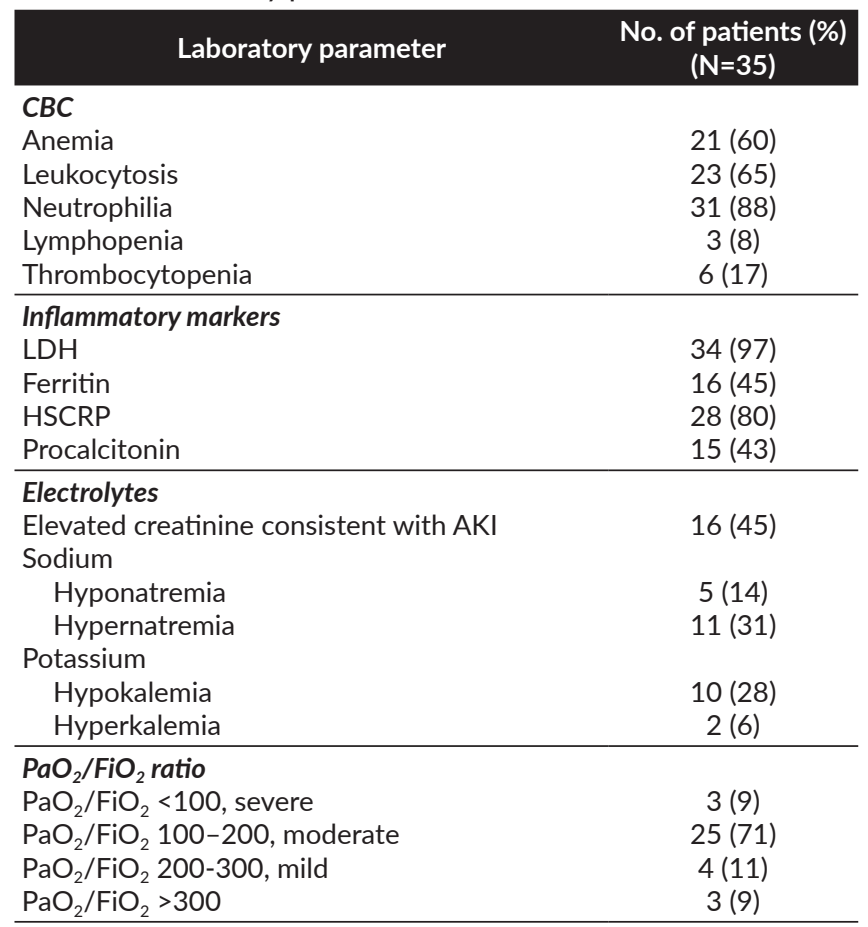

CBC, Complete blood count; LDH, Lactate dehydrogenase; HSCRP, high sensitivity C-reactive protein; AKI, Acute kidney injury; $\mathrm{PaO}_{2}$, Partial pressure of oxygen; $\mathrm{FiO}_{2}$, Fraction of inspired oxygen

bilateral consolidation but with no evidence of pulmonary embolism at time of study.

\section{Investigational Therapies}

During the initial months of admitting patients in the medical ICU, the investigational therapies offered to the patients were limited to the following: hydroxychloroquine,
Table 3. Radiologic Findings

\begin{tabular}{lc} 
Radiologic finding & $\begin{array}{c}\text { No. of patients } \\
\text { (N=35) }\end{array}$ \\
\hline Chest radiograph findings & 34 \\
Bilateral pneumonia & 1 \\
No active lung parenchymal infiltrates & 34 \\
\hline Characteristics of infiltrates on chest radiograph & 8 \\
Reticulonodular opacities & 7 \\
Alveolo-interstitial opacities & 6 \\
Inhomogenous opacities & 4 \\
Airspace opacities & 4 \\
Hazy opacities & 2 \\
\hline HRCT-chest & 2 \\
Bilateral ground glass opacities & 2 \\
Bilateral consolidation & \\
\hline
\end{tabular}

HRCT, High-resolution computed tomographic scan

chloroquine, lopinavir-ritonavir, tocilizumab, remdesivir, convalescent plasma therapy, and hemoperfusion. A total of 11 patients (31\%) received more than one investigational therapy, 10 (29\%) received one, and 14 (40\%) did not receive any. The most commonly administered drug was hydroxychloroquine (9 patients or 26\%), followed by chloroquine (7 or 20\%); hemoperfusion (6 or $17 \%$ ), lopinavirritonavir and tocilizumab (5 or 14\%). Less frequently given were convalescent plasma therapy (3 or 9\%) and remdesivir (2 or $6 \%)$.

\section{Respiratory Support and other ICU-level therapies}

All 35 patients required oxygen during their ICU stay, ranging from nasal cannula to a mechanical ventilator (Figure 3). Twenty-seven patients (77\%) needed high flow nasal cannula (HFNC) or intubation and were assessed to have acute respiratory failure, and was the most common reason for admission to the medical ICU. Patients were

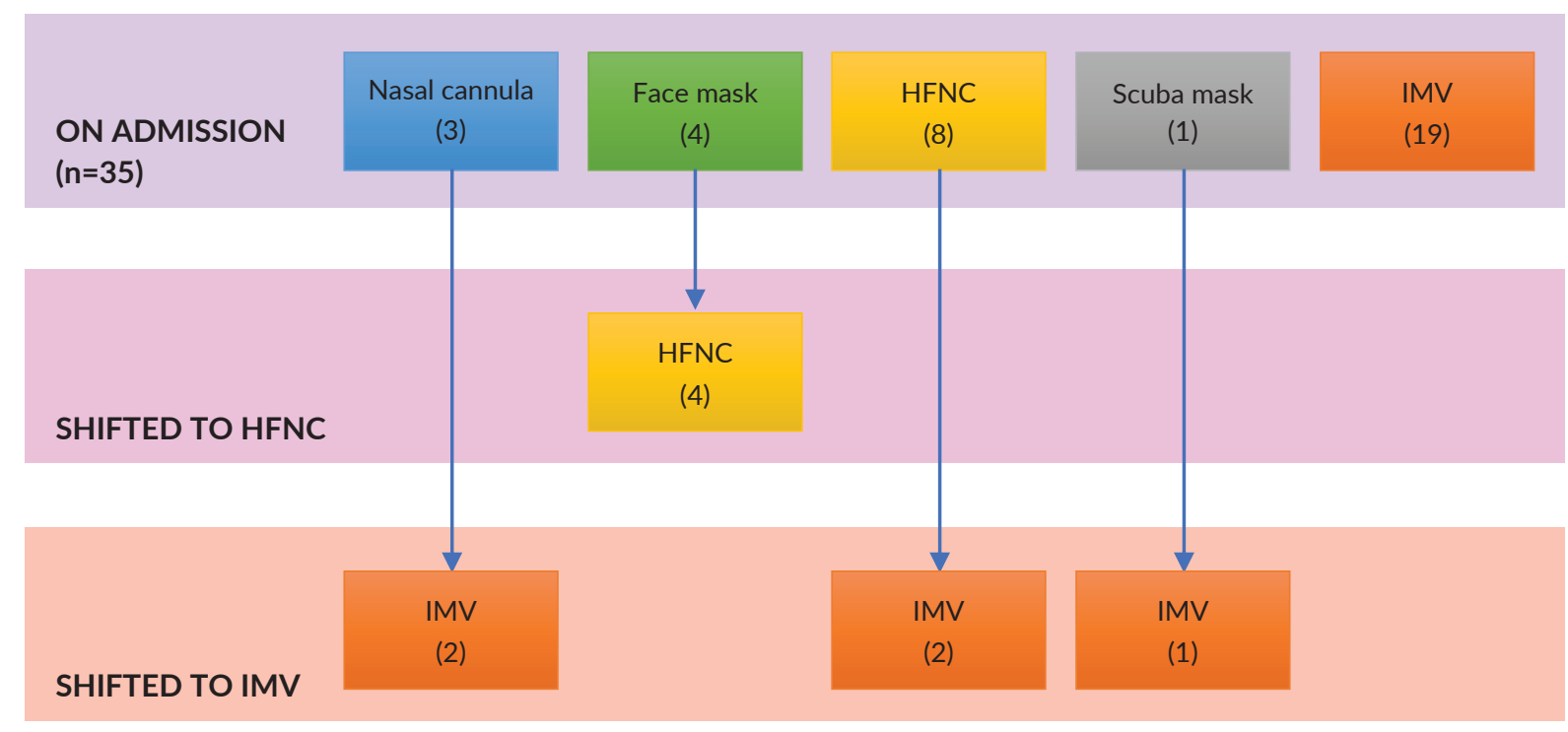

Figure 3. Oxygen support requirements of patients admitted in the medical ICU. Legend: HFNC - high flow nasal cannula, IMV - invasive mechanical ventilator. 
frequently intubated for persistent desaturations (peripheral $\mathrm{O}_{2}$ saturations $<90 \%$ ) and/or for decrease in sensorium, as seen in 13 patients. A total of 16 patients were received on non-invasive ventilation on ICU admission.

Three patients were received on nasal cannula $\left(\mathrm{FiO}_{2}\right.$ range $28-44 \%$ ), with two eventually being intubated: one was intubated for sudden decrease in sensorium, while the other had persistent tachypnea and desaturations. Both patients were commenced on $\mathrm{AC}-\mathrm{VC}$ mode at $\mathrm{FiO}_{2} 40 \%$.

A total of four patients were received on face mask $\left(\mathrm{FiO}_{2}\right.$ range $36-60 \%$ ) and were shifted to HFNC during their ICU stay. Initial $\mathrm{FiO}_{2}$ requirement on mechanical ventilation ranged from $60 \%$ to $95 \%$ for all four patients.

Eight patients were received on $\mathrm{HFNC}\left(\mathrm{FiO}_{2}\right.$ range 25\%-95\%); six patients were maintained on HFNC during their stay while two patients were intubated and was placed on $\mathrm{AC}$ mode with $\mathrm{FiO}_{2}$ at $80 \%$ and $100 \%$.

Scuba mask ventilation was tested on one patient (at $\mathrm{FiO}_{2} 80 \%$ ), but patient was intubated eventually for persistent desaturations and tachypnea. Invasive mechanical ventilation was commenced at $\mathrm{AC}-\mathrm{VC}$ mode with $\mathrm{FiO}_{2} 100 \%$.

In summary, from the 16 patients who were received at the medical ICU on non-invasive ventilation (nasal cannula, face mask, HFNC, scuba mask), eventual endotracheal intubation was done in 5 patients (Figure 3). Hence, a total of 24 patients out of the 35 patients admitted needed to be hooked to an invasive mechanical ventilator at some point during their ICU admission. Tracheostomy was done in one patient who was eventually discharged from the ICU.A total of five (5) patients were weaned from the mechanical ventilator.

Prone positioning was done for patients with severe ARDS with $\mathrm{P} / \mathrm{F}$ ratio $<150$ despite maximum oxygenation. Prone positioning entails keeping the patient on prone position for 16-20 hours to improve oxygenation status. For awake patients, self-proning was also recommended as long as the patient was cooperative and could tolerate the procedure. Proning was done in eight patients, over a mean duration of 2 days (range, 1 to 6 days). Out of these eight patients, self-proning was noted in two patients.

Vasopressor support to achieve MAP $>65$ was used for 15 patients (43\%), with a mean duration of 2.5 days (range, 1 to 6 days). Norepinephrine was used as the primary vasopressor in 14 patients; while one patient was received on dobutamine alone. Epinephrine and dobutamine, together with norepinephrine, was used in one patient.

\section{Outcomes}

The mean $( \pm \mathrm{SD})$ duration of admission was $6.85 \pm 6.6$ days (range, $<24$ hours to 36 days). The longest medical ICU admission was 36 days for a patient who eventually needed tracheostomy.

A total of 22 patients (63\%) died while 13 patients (37\%) were transferred out of the ICU. Of the 13 patients transferred out of the ICU, 12 patients were transferred out due to resolution of critical condition (e.g. hemodynamically
Table 4. Outcomes

\begin{tabular}{lc}
\multicolumn{1}{c}{ Outcome } & $\begin{array}{c}\text { No. of patients (\%) } \\
(\mathbf{N}=35)\end{array}$ \\
\hline $\begin{array}{l}\text { Primary outcome } \\
\text { Mortality }\end{array}$ & $22(63)$ \\
Discharged from ICU & $13(37)$ \\
\hline $\begin{array}{l}\text { Other outcomes } \\
\text { Liberated from mechanical ventilator / } \\
\quad \text { total patients on mechanical ventilator }\end{array}$ & $5 / 24$ \\
$\begin{array}{l}\text { Duration of admission, mean (range) in days } \\
\text { Duration of intubation, mean (range) in days }\end{array}$ & $\begin{array}{c}6.85 \pm 6.6(<24 \\
\text { hours to } 36 \text { days) } \\
10 \pm 16(2 \text { to } 38)\end{array}$ \\
\hline
\end{tabular}

stable, liberated from mechanical ventilator) and 1 patient was transferred out due to a poor prognosis and a "do not resuscitate" directive, which deemed the patient a poor candidate for ICU.

Out of the 24 patients who needed mechanical ventilation, a total of 5 patients were liberated from the machine with 4 patients being extubated and 1 patient undergoing tracheostomy. These five patients were discharged from the ICU. Mean $( \pm$ SD) duration of intubation for the five (5) extubated patients was $10 \pm 16$ days (range, 2 to 38 days). Mortality rate among patients who were intubated was $79 \%$.

\section{DISCUSSION}

A total of 35 critically ill patients were admitted between April 1 to May 31, 2020. Majority of patients were men and older than 60 years. Nearly all had at least one chronic medical illness, most common being hypertension (77\%) and diabetes (31\%). Fever was the most common symptom prior to hospital admission.

Most of the clinical characteristics (age, sex, common comorbid illness) of the patients are comparable with the early reports among critically ill patients from China, ${ }^{5}$ Italy, ${ }^{6}$ and New York. ${ }^{7}$ This study highlights how the disease can progress to the severe form among the elderly and patients with comorbid illness.

Anemia and leukocytosis were common in our set of patients. This is in contrast to current literature wherein the most common $\mathrm{CBC}$ abnormalities are lymphopenia and thrombocytopenia. ${ }^{8}$ Anemia is an independent risk factor for the severity of COVID-19, and is associated with increased mortality. ${ }^{9}$ The presence of leukocytosis might be due to superimposed bacterial infection in our cohort of patients, also reflected in the elevated procalcitonin levels seen in some patients. In one meta-analysis comprising of 56 studies, patients with severe disease in fact showed elevated levels of white blood cell count. ${ }^{10}$ This might imply that level of WBC count may also be a promising marker of severity.

Studies have suggested that monitoring inflammatory markers may predict disease severity and fatality as the disease can lead to a state of cytokine storm. ${ }^{11}$ Markers reported to be significantly associated with severe form of 
COVID-19 include LDH, CRP, ferritin, and procalcitonin. In our set of patients, most had elevated LDH and HSCRP upon admission, while elevation of ferritin and procalcitonin was found in $<50 \%$ of patients. In a study by Han et al, $\mathrm{LDH}$ was found to be an independent risk factor of severe COVID-19, showing highest correlation among other inflammatory markers with APACHE II score, SOFA score, and $\mathrm{PaO}_{2} / \mathrm{FiO}_{2}$ ratio. ${ }^{12}$ In the meta-analysis of $\mathrm{Zeng}$ et al., which was comprised of 16 studies, CRP and procalcitonin were positively correlated with the severity of COVID-19, among with other inflammatory markers, such as IL-6 and ESR. ${ }^{11}$ Ferritin, an acute phase reactant, is associated with poor prognosis and can be used as predictor for worsening clinical status among patients with COVID-19.13 More than half of the patients admitted at the ICU had moderate hypoxemia requiring oxygen supplementation. Santus et al, in a prospective observational multicentre study done in Italy, found that a moderate to severe impairment in PFR was associated with increased risk of in-hospital mortality. ${ }^{14}$

In terms of radiographic findings, bilateral pneumonia was present in almost all patients with reticulonodular opacities as the most common finding. In a study by Cozzi et al, the most common chest radiological features among COVID-19 patients were patchy or diffuse reticulonodular opacities and consolidation, with basal, peripheral, and bilateral predominance. ${ }^{15}$ HRCT was requested in two patients in whom a diagnosis of pulmonary embolism was considered. The incidence of thrombotic complications among patients with COVID-19 in the ICU was noted to be as high as $31 \%$ in the study of Klok et al. despite being on thromboprophylaxis. The disease itself develops a milieu of excessive inflammation and hypoxia further predisposing critically ill patients to thrombotic complications. ${ }^{16}$ The Philippine Society of Vascular Medicine supports the use of prophylactic anticoagulation in all moderate to critical COVID-19 patients in the absence of contraindications on its latest interim guidelines. ${ }^{17}$

Due to the novelty of the disease, no proven or approved treatments were available during the study. Numerous investigational therapies were offered to patients as compassionate use. During the first months of the pandemic, hydroxychloroquine (HCQ) and chloroquine (CQ) were two of the initial drugs proposed for COVID-19 treatment, as they were found to have in-vitro anti-viral activity against SARS-CoV and immune modulatory activity. ${ }^{18}$ Contraindications included prolonged QT interval (QTc > $500 \mathrm{~ms}$ ) and G6PD deficiency, hence the need to request for G6PD levels and 12L ECG tests for all patients with COVID-19. Multiple studies subsequently showed no benefit for the use of these drugs. In a systematic review from Singh et al. involving a total of 12 trials with over 8569 participants from all over the world, the authors concluded that HCQ has little or no effect on the risk of death and progression to mechanical ventilation among patients with COVID-19. ${ }^{19}$ Furthermore, adverse events were found more frequently among patients who took these drugs. Even in the local guidelines released by the Philippine Society for Microbiology and Infectious Diseases (PSMID), the use of HCQ and CQ as monotherapy or in combination with a macrolide among hospitalized patients is not recommended as of its latest updated version released July 20, 2020. ${ }^{20}$

Lopinavir-ritonavir, tocilizumab, and remdesivir were other investigational drugs offered to the patients, but their high cost made HCQ and CQ as the preferred drugs in our institution. The use of lopinavir-ritonavir combination was also found to have some activity in vitro against SARS$\mathrm{CoV}$. Early on, multiple studies showed some conflicting data but ultimately based on the preliminary results of RECOVERY and SOLIDARITY trials, both studies showed no beneficial effect among hospitalized patients with COVID-19. This was also the basis for the recommendation against using the drug for patients with COVID-19 in the latest PSMID guidelines. ${ }^{20}$

The use of tocilizumab, an IL-6 receptor blocker, may halt the cytokine storm associated with the severe form of COVID-19. A double-blind RCT showed that tocilizumab was not effective for preventing intubation or death in patients with moderate COVID-19, but some benefit or harm cannot be ruled out due to the wide confidence interval for efficacy. ${ }^{21}$ As for remdesivir, in the preliminary results of the Adaptive COVID-19 Treatment Trial (ACTT-1), a double-blind RCT comparing remdesivir with placebo, remdesivir was noted to be superior to placebo in shortening the time to recovery in adults hospitalized with COVID-19. ${ }^{22}$ The Infectious Disease Society of America (IDSA) in its latest guidelines still suggests the use of both drugs for severe or critical COVID-19 cases in addition to standard of care. ${ }^{23}$ Meanwhile, as per the latest PSMID guidelines, routine use has not yet been fully recommended for its use except in the context of clinical trial or compassionate use. ${ }^{20}$

In cases where investigational drugs could not be given due to contraindications or unavailability, convalescent plasma therapy and hemoperfusion were offered upon the recommendation of Hematology and Nephrology subspecialty services. Routine use of these therapies has not yet also been fully recommended in the local guidelines except in the context of compassionate use. ${ }^{20}$

All of the patients in this cohort required some form of oxygen support, with majority (69\%) ultimately hooked to an invasive mechanical ventilator. In the study of Xie et al. comprising of 733 critically ill adult patients with COVID-19, respiratory failure $\left(\mathrm{PaO}_{2} / \mathrm{FiO}_{2}\right.$ ratio less than $100 \mathrm{mmHg}$ ) was the most common type of organ failure. ${ }^{5}$ Similarly, the study by Bhatraju et al. showed that hypoxemic respiratory failure was the most common indication for ICU admission, ${ }^{24}$ as was found in our study. This high incidence of respiratory failure among patients with critical COVID-19 implies the need to increase the ICU capacity of hospitals and number of intensivists to care for the patients requiring ventilatory support and ICU level of care. 
The mortality rate in this cohort of patients (63\%) was similar to a retrospective multicenter study in China conducted during the first few months of the pandemic (January to February) (53.8\%). ${ }^{5}$ In another study consisting of patients in nine hospital ICUs across the Seattle region in Washington, with a time period of at least 14 days, mortality rate was found to be $50 \%$ between ICU day 1 and day 18 . All patients presented with acute hypoxemic respiratory failure. ${ }^{24}$ Older age, chronic cardiopulmonary diseases, and higher concentrations of interleukin-6 and D-dimer were suggested to be associated with in-hospital mortality in a prospective observational cohort study consisting of critically ill patients in two hospitals in New York City. ${ }^{7}$ All patients required oxygen support, either via invasive or non-invasive ventilation. The primary outcome in the New York study was in-hospital death, which was found to be $39 \%$ over the 28 day observation period.

The mortality rate in our set of patients was slightly higher compared to the reported rates in other studies which also included critically ill patients. It is important to note that the medical ICU admits all COVID-19 patients needing ICU level of care, regardless of the indication of admission. To emphasize, five of the patients included in this study came to the institution due to presentation of acute ischemic stroke, who eventually tested positive for the virus. The ICU does not limit admission to medical cases only, but also admits COVID-19 positive surgical patients where ICU admission was deemed necessary.

This study has several limitations. First, determining the risk factors of mortality was outside the scope of the study, which can provide key information in terms of who to prioritize for ICU admission. Second, although we did account for the investigational therapies offered to the patients, we did not collect data as to the clinical outcome related to the individual therapies received. Third, other medical complications arising during admission or trends of laboratory test results (i.e., inflammatory markers) could have been collected to help study the natural course of the disease. Fourth, our set of patients included those admitted to the medical ICU during the first two months of being a COVID-19 referral center, at a time when the medical resources were overwhelmed by the surge of cases of COVID-19 and when evidence on the use of therapies against SARS-CoV-2 was still limited. Further studies that will include the subsequent cohort of patients admitted to the ICU and in-depth analysis as to factors that increase risk of mortality is recommended.

\section{CONCLUSION}

A total of 35 critically ill COVID-19 patients were admitted to the medical ICU in UP-PGH during the first two months as a COVID-19 referral center. The baseline clinical characteristics of patients and findings of hypoxemic respiratory failure in this study are consistent with the reports from other studies focusing on critically ill patients.

\section{Statement of Authorship}

All authors participated in data collection and analysis, and approved the final version submitted.

\section{Author Disclosure:}

All authors declare no conflicts of interest in the study.

\section{Funding Source}

No funding was accepted in the making of this paper.

\section{REFERENCES}

1. Huang C, Wang Y, Li X, Ren L, Zhao J, Hu Y, et al. Clinical features of patients infected with 2019 novel coronavirus in Wuhan, China. Lancet. 2020 Feb 15; 395(10223):497-506.

2. Salva EP, Villarama JB, Lopez EB, Sayo AR, Villanueva AMG, Edwards T, et al. Epidemiological and clinical characteristics of patients with suspected COVID-19 admitted in Metro Manila, Philippines. Trop Med Health. 2020 Jul 7; 48:56.

3. Llaneta, C. UP-PGH begins operating as COVID-19 referral center. University of the Philippines [Internet], 2020 Apr 1 [cited October 31, 2020]; Available from https://www.up.edu.ph/up-pgh-beginsoperating-as-covid-19-referral-center/

4. Xie J, Wu W, Li S, Hu Y, Hu M, Li J, et al. Clinical characteristics and outcomes of critically ill patients with novel coronavirus infectious disease (COVID-19) in China: a retrospective multicenter study. Intensive Care Med. 2020 Oct; 46(10):1863-72.

5. Knaus WA, Draper EA, Wagner DP, Zimmerman JE. APACHE II: a severity of disease classification system. Crit Care Med. 1985 Oct; 13(10):818-29.

6. Grasselli G, Zangrillo A, Zanella A, Antonelli M, Cabrini L, Castelli A, et al. COVID-19 Lombardy ICU Network. Baseline characteristics and outcomes of 1591 patients infected with SARSCoV-2 admitted to ICUs of the Lombardy Region, Italy. JAMA. 2020 Apr 28; 323(16):1574-81.

7. Cummings MJ, Baldwin MR, Abrams D, Jacobson SD, Meyer BJ, Balough EM, et al. Epidemiology, clinical course, and outcomes of critically ill adults with COVID-19 in New York City: a prospective cohort study. Lancet. 2020 Jun 6; 395(10239):1763-70.

8. Pourbagheri-Sigaroodi A, Bashash D, Fateh F, Abolghasemi H. Laboratory findings in COVID-19 diagnosis and prognosis. Clin Chim Acta. 2020 Nov; 510:475-82.

9. Tao Z, Xu J, Chen W, Yang Z, Xu X, Liu L, et al. Anemia is associated with severe illness in COVID-19: a retrospective cohort study. J Med Virol. 2020 Aug 19:10.1002/jmv.26444.

10. Ji P, Zhu J, Zhong Z, Li H, Pang J, Li B, et al. Association of elevated inflammatory markers and severe COVID-19: a meta-analysis. Medicine (Baltimore). 2020 Nov 20; 99(47):e23315.

11. Zeng F, Huang Y, Guo Y, Yin M, Chen X, Xiao L, et al. Association of inflammatory markers with the severity of COVID-19: a metaanalysis. Int J Infect Dis. 2020 Jul;96:467-74.

12. Han Y, Zhang H, Mu S, Wei W, Jin C, Tong C, et al. Lactate dehydrogenase, an independent risk factor of severe COVID-19 patients: a retrospective and observational study. Aging (Albany NY). 2020 Jun 24; 12(12):11245-58.

13. Cheng L, Li H, Li L, Liu C, Yan S, Chen H, et al. Ferritin in the coronavirus disease 2019 (COVID-19): a systematic review and meta-analysis. J Clin Lab Anal. 2020 Oct; 34(10):e23618.

14. Santus P, Radovanovic D, Saderi L, Marino P, Cogliati C, De Filippis $\mathrm{G}$, et al. Severity of respiratory failure at admission and in-hospital mortality in patients with COVID-19: a prospective observational multicentre study. BMJ Open. 2020 Oct 10;10(10):e043651. 
15. Cozzi D, Albanesi M, Cavigli E, Moroni C, Bindi A, Luvarà S, et al. Chest X-ray in new Coronavirus Disease 2019 (COVID-19) infection: findings and correlation with clinical outcome. Radiol Med. 2020 Aug; 125(8):730-7.

16. Klok FA, Kruip MJHA, van der Meer NJM, Arbous MMS, Gommerse DAMPJ, Kant KM, et al. Incidence of thrombotic complications in critically ill ICU patients with COVID-19. Thromb Res. 2020 Jul; 191:145-7.

17. Philippine Society of Vascular Medicine. PSVM Interim Guidelines on Role of Anticoagulation in COVID-19: Recommendations on its Use, (an UPDATE). July 2020.

18. Meyerowitz EA, Vannier AGL, Friesen MGN, Schoenfeld S, Gelfand JA, Callahan MV, et al. Rethinking the role of hydroxychloroquine in the treatment of COVID-19. FASEB J. 2020 May; 34(5):6027-37.

19. Singh B, Ryan H, Kredo T, Chaplin M, Fletcher T. Chloroquine or hydroxychloroquine for prevention and treatment of COVID-19. Cochrane Database Syst Rev. 2021 Feb 12; 2:CD013587. doi: 10.1002/14651858.CD013587.pub2..

20. Philippine Society for Microbiology and Infectious Diseases. Interim Guidance on the Clinical Management of Adult Patients with Suspected or Confirmed COVID-19 Infection (Version 3.1). [Internet] July 20, 2020. [cited 2021 January] Available from https://www.psmid.org/interim-management-guidelines-for-covid19-version-3-1/.
21. Stone JH, Frigault MJ, Serling-Boyd NJ, Fernandes AD, Harvey L, Foulkeset AS, et al. Efficacy of tocilizumab in patients hospitalized with COVID-19. N Engl J Med. 2020 Dec 10; 383(24):2333-44. doi: 10.1056/NEJMoa2028836.

22. Beigel JH, Tomashek KM, Dodd LE, Mehta AK, Zingman BS, Kalil AC, et al. Remdesivir for the treatment of COVID-19 Final Report. N Engl J Med. 2020 Nov 5;383(19):1813-1826. doi: 10.1056/NEJMoa2007764..

23. Bhimraj A, Morgan RL, Shumaker AH, Lavergne V, Baden L, Cheng VC, et al. Infectious Diseases Society of America Guidelines on the Treatment and Management of Patients with COVID-19. Clin Infect Dis. 2020 Apr 27:ciaa478. doi: 10.1093/cid/ciaa478.

24. Bhatraju PK, Ghassemieh BJ, Nichols M, Kim R, Jerome KR, Nalla AK, et al. COVID-19 in critically ill patients in the Seattle Region Case series. N Engl J Med. 2020 May 21; 382(21):2012-22. 